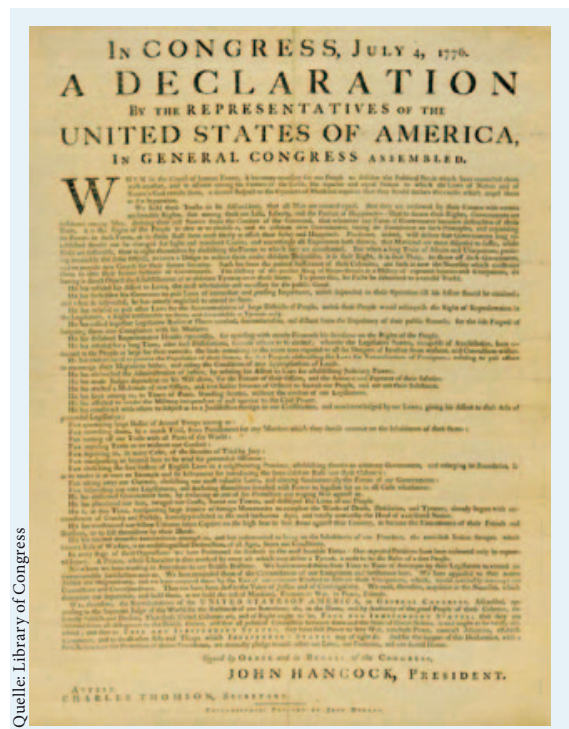

\section{Psychische Erkrankungen}

Die Bestsellerlisten sind voll der Ratgeber über die Wege zum Glück. Seit den vorsokratischen Philosophen gilt das glückliche Leben als Ergebnis der inneren Verfassung und Seele; es zu ermöglichen sah schon im 12. Jahrhundert der islamische Arzt und Philosoph Ibn Ruschd als Aufgabe des Staates, ebenso wie 600 Jahre später die Gründerväter der USA. Für sie war "the pursuit of happiness» in der Unabhängigkeitserklärung, die Sie oben sehen, das höchste Gut. Doch des Menschen Psyche leidet - entweder unter selbst gemachtem Stress oder unter Erkrankungen, die einen organischen Ursprung haben. Was es mit den Problemen auf sich hat, die durch Anspannung im Beruf und im modernen Leben entstehen, lesen Sie hier gleich nebenan. Wie man mit Johanniskraut Depressionen bekämpft, steht auf der Seite 264, und in unserem Expertengespräch ab der Seite 260 erfahren Sie, was führende Schweizer Exponenten der Ganzheitsmedizin empfehlen, um Patienten mit seelischen Erkrankungen zu behandeln.

\title{
Aufbauhilfe für Businesskrieger und Multitasker
}

Gegen Burnout hilft eigentlich nur die Vollbremsung. Raus aus dem Stress und abschalten. Methoden der Ganzheitsmedizin helfen, wieder auf den Boden zu kommen, ruhig zu werden und das Verhalten zu ändern.

Was in den 1970er-Jahren von dem amerikanischen Psychologen Herbert Freudenberger als Phänomen in den heilenden Berufen beschrieben wurde, hat sich mittlerweile zur Massenerscheinung entwickelt. Getrieben von Stress, Hektik, Überforderung und Multitasking geraten immer mehr Menschen an den Rand der totalen Erschöpfung: Burnout. Keine Kraft, keine Energie, keine Lust mehr an der Arbeit. Jeden vierten oder fünften Berufstätigen erwischt das Burnout. Allein der wirtschaftliche Schaden ist astronomisch: Auf knapp 4,2 Milliarden Franken beziffert das Staatssekretariat für Wirtschaft (Seco) den jährlich durch Stress entstehenden Schaden. Auch Frauen, die sich über eine lange Zeit um pflegebedürftige Angehörige kümmern, laufen oft in ein Burnout. Aus der gesellschaftlichen Entwicklung, die für die Verbreitung des Burnout verantwortlich ist, gibt es nur einen Ausweg: Die Vollbremsung und die Veränderung der eigenen Einstellung zur Arbeit und zum Leben. «Es braucht ein anderes Verhalten des Einzelnen, um das Burnout zu kurieren und einen Rückfall zu verhindern», sagt die Ärztin und Psychotherapeutin Dagmar Wemmer, die in ihrer Praxis in Mosnang mit Methoden der Naturheilkunde arbeitet und auch pflanzliche Mittel zur Behandlung von Symptomen des Burnout einsetzt.
Modernes Leben wird immer stressiger Für viele war der rasante Anstieg der Burnout-Fälle in den letzten ein bis zwei Jahrzehnten eher ein Zeichen dafür, dass eine neue Modediagnose die Runde macht, als dass ein wirkliches, neues medizinisches Problem vorliegt. Mittlerweile ist die Diagnose etabliert. «Vielen Menschen fällt es auch leichter, mit der Diagnose Burnout umzugehen, als mit dem Stigma Depression zu leben», sagt Dagmar Wemmer. Manche Symptome des Burnout gleichen tatsächlich - vor allem bei schwereren Burnout-Fällen - denen einer Depression. Dazu gehören Schlafstörungen, das ständige Kreisen der Gedanken, die Gefühle der Erschöpfung, der Niedergeschlagenheit und fehlenden Zuversicht bis hin $\mathrm{zu}$ totaler Verzweiflung und Gedanken an einen Suizid. «Für die Therapie ist es aber sehr wichtig, zu wissen, ob eine Depression oder ein Burnout vorliegt. Während der Mensch mit Burnout sich meist überschätzt und seine Leistungen immer noch mehr versucht anzutreiben - z.B. aus dem Wunsch nach Anerkennung oder auch aus der fehlenden Fähigkeit, sich abzugrenzen -, neigt der Depressive zu einer permanenten Unterschätzung seiner Fähigkeiten und wird von Selbstzweifeln geplagt», sagt sie. Drei Dinge kennzeichnen einen Patienten, der an einem Burnout leidet: Zum Ersten eine emotionale und körperliche Erschöpfung. Zweitens Zynismus: Menschen, die vorher empathisch und leistungsbereit waren,

\section{KARGER}




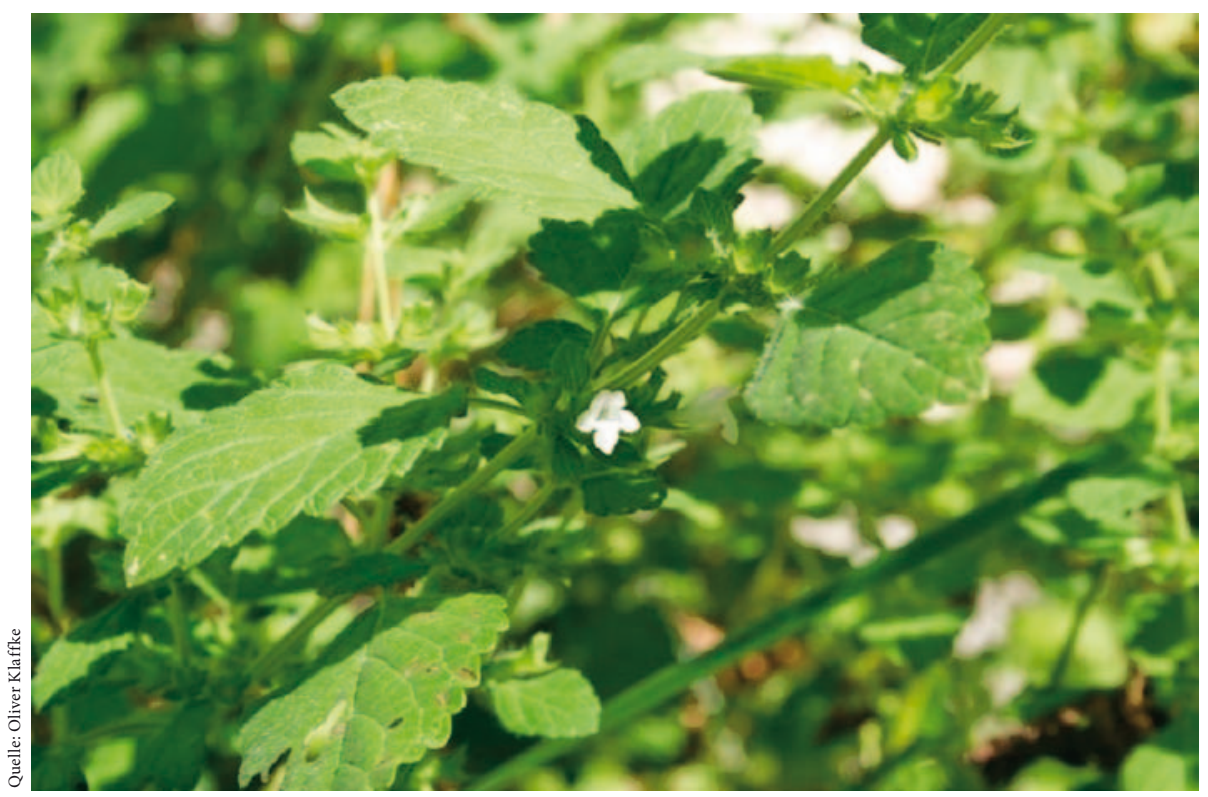

Abb. 1. Melissa officinalis (Zitronenmelisse).

verkehren sich ins Gegenteil und werden zynisch, entwertend gegenüber der Arbeit, ungeduldig bis aggressiv. Und drittens bringen sie am Schluss die Leistung nicht mehr, die von ihnen erwartet wird. «Sie sind nicht mehr zuverlässig, werden vergesslich, und die unerledigten Stapel auf dem Schreibtisch beginnen sich zu türmen», sagt der Psychiater und ehemalige Chefarzt Psychiatrische Dienste Aargau, Roman Vogt. «Stellen Sie sich Pflegende in einem Altersheim vor, die ausgebrannt sind: Sie ertragen die verwirrten, verlangsamten und betagten Menschen nicht mehr.» Sie reagieren mit Zurechtweisungen - und bis zu Tätlichkeiten aus Frustration ist es dann nur noch ein kurzer Weg.

\section{Die Arbeit macht krank}

Die meisten Ärzte sind sich einig, dass die Ursachen des Burnout in der heutigen Arbeitswelt liegen. Viele Menschen stehen beruflich an vielen Fronten unter sehr grosser Anspannung. Zum einen ist es der Zeitdruck, der mittlerweile herrscht. Ein gesundes Arbeitstempo ist fast nirgends mehr möglich; es muss alles am besten schon gestern erledigt sein, was heute als Auftrag auf den Tisch oder meistens auf den Bildschirm kommt. Zum wicklung aus. Im Marktwettbewerb müssen wir ständig die Besten sein. «Was wirklich krank macht, ist nicht Informationsüberflutung, sondern der neoliberale Druck mit seinen unmöglichen Arbeitsbedingungen», meint Berardi. Das führe zu permanentem Aufmerksamkeitsstress, für Affektivität bleibe immer weniger Zeit. «Wenn wir Souveränität erlangen wollen, müssen wir unbedingt wieder Herr unserer Zeit werden», meint Lovink. Man müsse die Echtzeitstrategien von Google und Twitter bekämpfen und wieder längere Arbeitssequenzen entwickeln. Nach Slow Food sei nun endlich Slow Communication notwendig.

\section{Streben nach Flexibilität ist schuld}

In einem Aufsatz in der Zeitschrift Schweizerisches Medizinisches Forum (2010;10:148-151) beleuchtet Toni Brühlmann von der Privatklinik Hohenegg in Meilen den Zusammenhang zwischen Burnout und der gesellschaftlichen Situation. Der Charakter des Menschen werde durch die wirtschaftliche Entwicklung untergraben, wie es etwa der Soziologe Richard Sennett schon 1998 in seinem Buch «Der flexible Mensch» gezeigt habe: Nicht mehr Verbindlichkeit und Tiefgang zählten, sondern die flexible Oberflächlichkeit und das ziellose Dahintreiben. «Das Beständige und Haltgebende wird fortlaufend zerstört», schreibt Brühlmann. Eine Fragmentation sei die Folge und die Stärke der Bindungen zwischen den Menschen nehme ab. Stattdessen ist der Mensch auf sich alleine gestellt und begreift sich zunehmend als Ich AG. Die Konsequenzen, so Brühlmann, habe schon der französische Soziologe Alain Ehrenberg 1998 in seinem Buch «Das erschöpfte Selbst» skizziert: Die Depression als die zeittypische Krankheit. «In der hektischen Leistungsgesellschaft hat man sich immer neu zu definieren, um konkurrenzfähig zu bleiben», meint Brühlmann. Ein Prozess, der auslaugt und erschöpft. 
Enorm gefährdet sind Männer und Frauen, die in Führungspositionen stecken. Allein durch die enormen Arbeitspensen von nicht selten 16, 18 Stunden täglich sind sie besonders anfällig, unter dem Druck zusammenzubrechen: Sie haben eine anspruchsvolle Tätigkeit, die Vorgaben der Unternehmensleitung zu erfüllen und sich längst abgewöhnt, sich von der eigenen Arbeit zu distanzieren. Es sind bestimmte Persönlichkeitsmerkmale, die das Burnout fördern, und solche, die im modernen Arbeitsleben besonders gefragt sind: Pflichtbewusstsein, Gewissenhaftigkeit und Leistungswille. «Von der Persönlichkeit her sind es oft Menschen, die nicht Nein sagen können, sich keine Grenzen setzen und ständig aktiv sind", sagt Dagmar Wemmer. Solche, die die Arbeit und den Stress in alle anderen Bereiche des Lebens eindringen lassen, weil die Trennung von Beruf und Privatleben längst nicht mehr vorhanden ist. Die andere besonders gefährdete Gruppe sind Menschen, die zu Hause Betreuungspflichten wahrnehmen; seien es Frauen, die ihre betagten Eltern pflegen, seien es junge berufstätige Mütter - sie werden von ihrem Pflichtgefühl fast erdrückt.

\section{Verhalten muss sich ändern}

«Die Therapie bei einem Burnout muss auf der Verhaltensebene ansetzen», sagt Dagmar Wemmer. Beim Weg aus dem Ausgebranntsein geht es nicht einfach um ein Time-out, sondern darum, in einem ersten Innehalten sein Verhalten und die Umstände zu erkennen, die zum Burnout geführt haben, und mit professioneller Hilfe eine Änderung herbeizuführen. Das Verhalten, das zum Burnout geführt hat, müsse Schritt für Schritt in eine neue Richtung gelenkt werden, um einen Rückfall zu verhindern. Wichtig sei auch, die Symptome zu behandeln, damit die Betroffenen wieder die Möglichkeit hätten, sich wieder zu fangen. «Entscheidend ist, dass die Betroffenen, welche an Schlafstörungen leiden, wieder schlafen und sich entspannen lernen, um sich zu erholen.» Aus der Phytotherapie (siehe Kasten) bieten sich Johanniskraut zur Depressionsbehandlung, phytotherapeutische Entspannungspräparate, milde pflanzliche Schlafmittel und Adaptogene zur Behandlung an. «Die Adaptogene darf man aber nicht in der Absicht geben, dass der Patient sein Verhalten unverändert fortsetzt und nur etwas belastbarer ist», sagt sie. Die Verhaltensänderung ist unabdingbar. In den meisten Fällen sei eine Psychotherapie erforderlich; in leichten Fällen könne bereits ein Coach oder das Gespräch mit einem Vertrauten ausserhalb der eigenen Familie den Betroffenen ein gutes Stück weiterbringen. Die Verbesserung der Körperwahrnehmung ist enorm wichtig, denn viele Betroffenen «blenden» das eigene Körpergefühl aus, reagieren weder auf frühe Warnzeichnen noch nehmen sie häufig Magenschmerzen, Herzbeschwerden oder andere somatische Beschwerden ernst. Meditation oder andere Entspannungstechniken stellen eine wichtige Säule der Burnout-Behandlung dar. Auch phytotherapeutische pflegerische Anwendungen mit Wickeln, Auflagen, Einreibungen oder Fussbädern können diesen Prozess unterstützen.

\section{Arbeit ist nicht alles}

Wichtig ist die Prävention des Burnout, und dabei spielt das eigene Verhalten die entscheidende Rolle. «Es ist enorm wichtig, dass wir Arbeit nicht als das Alleinseligmachende anschauen, sondern erstens den Ausgleich pflegen und zweitens die Arbeit begrenzen», sagt Roman Vogt. Es sei wichtig, Fehler zuzulassen und auch einmal statt 100 nur 80\% abzuliefern. «Man muss Nein sagen und den Ärger der Vorgesetzten oder Kollegen in Kauf nehmen können; deshalb stürzt die Welt nicht ein», sagt er. Und die «Macho-Riege» der Manager müsse lernen, dass es neben beruflichem Erfolg noch andere Werte gibt. «Leben zum Beispiel.» (rfi)

\section{Phytotherapie bei Burnout}

Als milde Tranquilizer:

- Lavendel (Lavandula angustifolia): Lavendelblütentee, Lavendelölauflagen, Lavendeleinreibungen, Lavendelbad.

- Melisse (Melissa officinalis): Wie Lavendel, aber schwächer wirksam.

- Passionsblume (Passiflora incarnata): Fertigarzneimittel.

Als milde Sedativa:

- Baldrianwurzel (Valeriana officinalis)/Hopfenzapfen (Humulus lupulus) als Fertigarzneimittel (Kombination war in Studien wirksamer als die Einzelsubstanzen).

Als Antidepressivum bei schwererem Burnout:

- Johanniskraut (Hypericum perforatum) als Fertigarzneimittel.

Als Adaptogene bei unvermeidbaren Belastungen:

- Ginsengwurzel (Panax ginseng): Fertigarzneimittel - als Tonikum seit Langem zugelassen; gut auch mit Ginkgo kombinierbar (Steigerung der geistigen Leistungsfähigkeit).

- Taigawurzel (Eleutherococcus senticosus): Seit 2009 als Arzneimittel (Tonikum) in der Schweiz zugelassen.

- Rosenwurz (Rhodiola rosea): Seit Ende Mai 2010 in der Schweiz als Arzneimittel (Tonikum) zugelassen. Es wurden neben stimulierenden und adaptogenen Eigenschaften auch kardioprotektive, antidepressive und anxiolytische Eigenschaften nachgewiesen; die Studien von 2009 werden qualitativ jedoch hinterfragt. 